\title{
A geometric application to the third-order recurrence relations for sequences
}

\author{
Calin M. Agut and Ionna E. Agut
}

Abstract. Using a third-order linear homogeneous recurrence relation with constant coefficients, it is found a limit-point of a sequence of affixes in plane. Starting from a classic geometric problem, an application is so created and few more nice properties are found and described.

Key words and phrases: affixes, recurrent sequences, generalization, specialization, similarity, limits.

ZDM Subject Classification: B40, B50, D30, F50, G10, G40, I30.

\section{Introduction}

We have found an interesting geometric application for a special kind of recurrently defines sequences, starting form a classic problem in a regular course of Geometry:

Problem 1. In an arbitrary triangle $A B C$ we consider the midpoints $M_{1} \in(A B), M_{2} \in(B C), M_{3} \in\left(C M_{1}\right), M_{4} \in\left(M_{1} M_{2}\right)$, etc. of the line segments $(A B),(B C),\left(C M_{1}\right),\left(M_{1} M_{2}\right)$, etc., respectively. If we continue to build such points $M_{n}$, what will be the location of $M_{n}$ when $n \rightarrow \infty$ ?

If we try to build the points we define in fact a sequence $\left(M_{n}\right)$, where each point $M_{n}$ has two coordinates whose are linearly related with some of the previous constructed coordinates. In this way we approach to the theory of sequences 
defined by a recurrent relation, so that the general term will have an explicit expression which leads us to a possible limit when $n \rightarrow \infty$.

The theory of sequences is more related to the topic and curricula in Mathematics Analysis or Calculus rather than to Geometry. Moreover, the study of the third-order linear homogeneous recurrence relations with constant coefficients (which will occur) is not very often contained not in the high school topic nor even in the college undergraduate courses. Our intention here is to connect such geometrical exercise with such kind of recursively defined sequences, using aspects related to mathematical analysis, complex analysis and geometry. Also we will use the concepts of generalization and specialization, as a teaching goal and strategy for mathematics classes, at the high school or college level. The work presented here will limit to the two-dimensional space.

If for sequences of real numbers we have a very clear representation on the number-line, in the case of sequences of complex numbers we need the entire 2-D plane. In this way, we will connect the study with 2-D Coordinate Geometry.

The special kind of recurrent sequences $\left(x_{n}\right)_{n \geqslant 0}$ used here is so called a thirdorder linear homogeneous recursively defined sequence with constant coefficients:

$$
x_{n}=\lambda x_{n-1}+\mu x_{n-2}+\eta x_{n-3} .
$$

The present paper will contain an answer for Problem 1 using the above sequences, but first our intention is to work with the general case considering not just the midpoints of the segments. We will obtain a generalization of the Problem 1 with different initial conditions (Propositions 1, 3 and 4) and then, in order to give an answer for it, we will go backward with a Specialization (Proposition 2).

Moreover, in the general case there will be stated some interesting properties about the limit point(s) (Propositions 5, 6 and 7).

We denote an "initial" triangle by $Z_{0} Z_{1} Z_{2}$ and we choose a point $Z_{3}$ on the side $Z_{0} Z_{1}$ such that $\frac{\left|Z_{0} Z_{3}\right|}{\left|Z_{0} Z_{1}\right|}=\frac{1}{m}$ (here $\left|Z_{0} Z_{1}\right|$ denotes the length of the side $Z_{0} Z_{1}$ ) where $m>1$ is a real number.

Next, we choose the point $Z_{4} \in\left(Z_{1} Z_{2}\right)$, such that $\frac{\left|Z_{1} Z_{4}\right|}{\left|Z_{1} Z_{2}\right|}=\frac{1}{m}$, the point $Z_{5} \in\left(Z_{2} Z_{3}\right)$, such that $\frac{\left|Z_{2} Z_{5}\right|}{\left|Z_{2} Z_{3}\right|}=\frac{1}{m}$, the point $Z_{6} \in\left(Z_{3} Z_{4}\right)$, such that $\frac{\left|Z_{3} Z_{6}\right|}{\left|Z_{3} Z_{4}\right|}=\frac{1}{m}$, and so forth, obtaining a sequence of points, denoted by $\left(Z_{n}\right)_{n \geqslant 0}$.

Definition 1. Because the point $Z_{3}$ is chosen on the opposite side to $Z_{2}$, we tell that the starting point in our construction is $Z_{2}$.

It is easy to see that we may consider as starting points the vertices $Z_{0}$ and $Z_{1}$, following the same process in obtaining sequences of complex numbers. We will 


$$
\text { "tmcs-agut" — 2010/11/14 — 22:36 — page } 289 \text { — \#3 }
$$

see that all of them converge to "limit-point", an affix of an interior point of given triangle.

Overall we will have to work with three different sequences and three different limit points. These three limit points, connected, will form a triangle which is similar to the triangle $Z_{0} Z_{1} Z_{3}$, for any value of $m>1$.

\section{Preliminaries}

The work needed and presented here will mix the methods of Calculus, Coordinate Geometry and the Theory of Difference Equations. Here we take it for granted that the Reader is familiar with the notion of complex sequences.

\subsection{Recursive sequences}

A particular case of sequences of numbers (real or complex) is given by recursively expression, when a term of the sequence is related to one or more of its previous terms (regarding the rank). We recall here one class of the recurrent sequences:

Definition 2. Let $\lambda, \mu, \eta$ be arbitrary real numbers. The sequence $\left(x_{n}\right)_{n \geqslant 0}$ is called a third order linear homogeneous recursively defined sequence if for all $n \geqslant 0$ the difference equation

$$
x_{n+3}=\lambda x_{n+2}+\mu x_{n+1}+\eta x_{n},
$$

is fulfilled.

From this definition it can be seen that recursive sequences are special difference equations. This remark is very useful, since the theory of difference equations has an extensive literature (see [1], [2], [6]).

It is well-known that for equation (2.1) we can associate an algebraic equation

$$
w^{3}-\lambda w^{2}-\mu w-\eta=0,
$$

which is so called characteristic equation of (2.1).

Furthermore, it will also be utilized that the solutions of (2.1) can be determined with the aid of the roots of the algebraic equation (2.2). Let us suppose 
that the equation (2.2) has the roots $w_{1}, w_{2}$, and $w_{3}$, where $w_{i} \in \mathbb{C}, i=1,2,3$. Then, and only then,

$$
x_{n}=A_{1}\left(w_{1}\right)^{n}+A_{2}\left(w_{2}\right)^{n}+A_{3}\left(w_{3}\right)^{n},
$$

holds for all $n \geqslant 0$, where $A_{1}, A_{2}, A_{3}$ are arbitrary real constants.

We remark that concerning the roots of equation (2.2) the following two cases are possible:

Case A: In the first case suppose that $w_{1} \in \mathbb{R}$ and $w_{2}, w_{3} \in \mathbb{C}$. It is obvious that in this case $w_{2}=\overline{w_{3}}$. Furthermore, in the case that

$$
w_{2}=\rho(\cos \theta+i \sin \theta) \text { and } w_{3}=\rho(\cos \theta-i \sin \theta),
$$

then equation (2.3) can be written as:

$$
x_{n}=A_{1}(r)^{n}+A_{2} \rho^{n}(\cos (n \theta)+i \sin (n \theta))+A_{3} \rho^{n}(\cos (n \theta)-i \sin (n \theta)) .
$$

Case B: The other possibility is that equation (2.2) has only real roots. In this case equation (2.3) is unchanged. We know however, that the numbers $w_{1}$, $w_{2}$, and $w_{3}$ are real numbers.

Using the preceding results on equation (2.2) the following two Lemmata can be easily derived:

Lemma 1. If the characteristic equation (2.2) has one real root $r=1$ and two complex roots, such that $\rho \in(-1,1)$, then $\lim _{n \rightarrow \infty} x_{n}=A_{1}$.

Lemma 2. If the characteristic equation (2.2) has three real roots, such that $w_{1}=r=1$ and $w_{2,3} \in(-1,1)$, then $\lim _{n \rightarrow \infty} x_{n}=A_{1}$.

\subsection{Coordinate geometry}

In Coordinate Geometry in 2-D plane each point has assigned a pair of numbers, called coordinates.

If we consider two distinct points in $x O y$ plane, let us name them $A\left(x_{A}, y_{A}\right)$ and $B\left(x_{B}, y_{B}\right)$. The equation of the line $A B$ has the form

$$
(A B): y=\frac{y_{B}-y_{A}}{x_{B}-x_{A}} x+b,
$$

where $m=\frac{y_{B}-y_{A}}{x_{B}-x_{A}}$ is the slope and $b$ is the $y$-intercept. 


$$
\text { "tmcs-agut" — 2010/11/14 — 22:36 — page } 291 \text { — \#5 }
$$

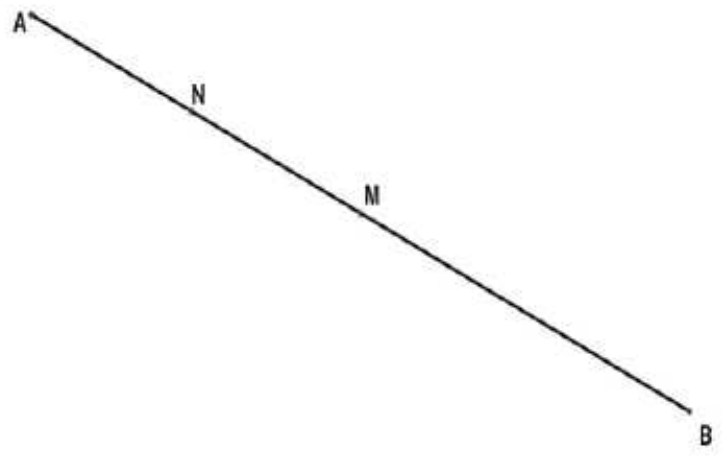

Figure 1

If $M\left(x_{M}, y_{M}\right)$ is the midpoint of the line segment $A B$, then its coordinates will have the expressions

$$
x_{M}=\frac{x_{A}+x_{B}}{2}, \quad y_{M}=\frac{y_{A}+y_{B}}{2} .
$$

More general, if $N\left(x_{N}, y_{N}\right)$ is an interior point of the line segment $A B$ such that $\frac{|A N|}{|A B|}=\frac{1}{n}$ (with $n>1$ ), where $|A B|$ denotes the length of the segment $A B$, then the coordinates of $N$ will have the expressions:

$$
x_{N}=\frac{n-1}{n} x_{A}+\frac{1}{n} x_{B}, \quad y_{N}=\frac{n-1}{n} y_{A}+\frac{1}{n} y_{B} .
$$

\section{Sequence of affixes and limit-point}

\subsection{Applying the third-order linear homogeneous recurrence}

In this section of the paper we will investigate Problem 1, presented at the beginning. Results concerning Problem 1 will be presented as a corollary of a more general problem. In this case the initial triangle will be termed $Z_{0} Z_{1} Z_{2}$ and we will work not with midpoints $M_{i}$, but points $Z_{i}$ with the property $Z_{i} \in Z_{i-3} Z_{i-2}$ and

$$
\frac{\left|Z_{i-3} Z_{i}\right|}{\left|Z_{i-3} Z_{i-2}\right|}=\frac{1}{m}
$$

for all $i \geq 3$, where $m>1$ is a fixed real parameter. 
Consider a triangle $Z_{0} Z_{1} Z_{2}$ and choose a point $Z_{3}$ in the interior of the segment $Z_{0} Z_{1}$ such that

$$
\frac{\left|Z_{0} Z_{3}\right|}{\left|Z_{0} Z_{1}\right|}=\frac{1}{m}
$$

holds, where $\left|Z_{0} Z_{1}\right|$ denotes the length of the segment $Z_{0} Z_{1}$.

Next, we choose the point $Z_{4} \in\left(Z_{1} Z_{2}\right)$, such that $\frac{\left|Z_{1} Z_{4}\right|}{\left|Z_{1} Z_{2}\right|}=\frac{1}{m}$, the point $Z_{5} \in\left(Z_{2} Z_{3}\right)$, such that $\frac{\left|Z_{2} Z_{5}\right|}{\left|Z_{2} Z_{3}\right|}=\frac{1}{m}$, the point $Z_{6} \in\left(Z_{3} Z_{4}\right)$, such that $\frac{\left|Z_{3} Z_{6}\right|}{\left|Z_{3} Z_{4}\right|}=\frac{1}{m}$, and so forth, obtaining a sequence of points, denoting it by $\left(Z_{n}\right)_{n \geqslant 0}$.

Our starting point here is considered to be $Z_{2}$.

For simplicity, let us consider $Z_{0} Z_{1} Z_{2}$ be an arbitrary triangle (see Figure 1), with the vertices in Cartesian Coordinate System $Z_{0}(0,0), Z_{1}(a, 0)$ and $Z_{2}(b, t)$, where $a>0, b>0$ and $t>0$ are real numbers, and for convenience, $a \geq b$. Also, we denote in general by $Z_{n}\left(x_{n}, y_{n}\right)$ the obtained points, for $n \geq 3$.

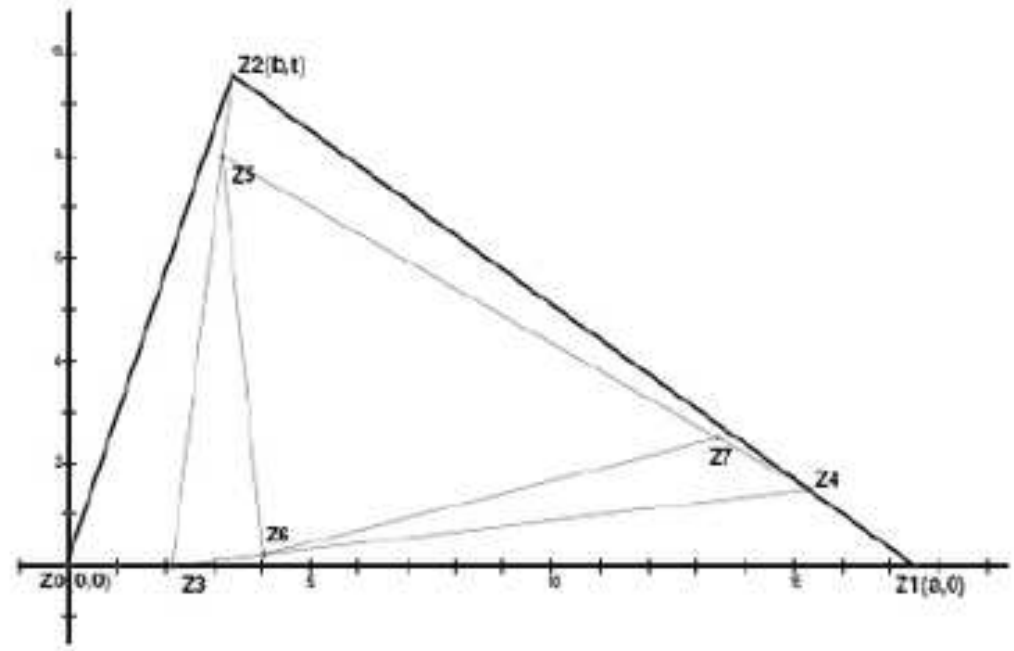

Figure 2

In this situation we just created a sequence of points, denoted by $\left(Z_{n}\left(x_{n}, y_{n}\right)\right)_{n \geq 0}$. Obviously, we have the initial conditions: $x_{0}=0, x_{1}=a, x_{2}=b, y_{0}=y_{1}=0$, and $y_{2}=t$.

Moreover, we have created a sequence of affixes denoted by $\left(z_{n}\right)_{n \geq 0}$, where $z_{n}=x_{n}+i y_{n}$.

The sequences $\left(x_{n}\right)_{n \geq 0}$ and $\left(y_{n}\right)_{n \geq 0}$ are sequences of real numbers. 
In the next part of the section we will study the convergence of the sequence $\left(z_{n}\right)_{n \geq 0}$, and, if it converges, we will find the limit point.

Since $Z_{3} \in\left(Z_{0} Z_{1}\right)$ such that $\frac{\left|Z_{0} Z_{3}\right|}{\left|Z_{0} Z_{1}\right|}=\frac{1}{\mathrm{~m}}$, then using the relations (2.6) we can write

$$
x_{3}=\frac{m-1}{m} x_{0}+\frac{1}{m} x_{1}, \quad \text { and } \quad y_{3}=\frac{m-1}{m} y_{0}+\frac{1}{m} y_{1} .
$$

Continuing the process, we can give the recursive form for the terms of the sequences $\left(x_{n}\right)_{n \geqslant 0}$ and $\left(y_{n}\right)_{n \geqslant 0}$ as follows:

$$
\begin{aligned}
& x_{n+3}=\frac{1}{m} x_{n+1}+\frac{m-1}{m} x_{n}, \\
& y_{n+3}=\frac{1}{m} y_{n+1}+\frac{m-1}{m} y_{n},
\end{aligned}
$$

for any integer $n \geqslant 0$ and any $m>1$.

For simplicity, because (3.1) and (3.2) have the same coefficients, we may consider for both the following characteristic equation:

$$
m w^{3}-w-m+1=0 .
$$

The equation (3.3) has $w=1$ the real solution and may be factored:

$$
(w-1)\left(m w^{2}+m w+m-1\right)=0 .
$$

The second factor of (3.4) is a quadratic equation

$$
m w^{2}+m w+m-1=0,
$$

for which the discriminant $\Delta$ has the expression

$$
\Delta=\frac{-m \pm \sqrt{4 m-3 m^{2}}}{2 m} .
$$

Since $m>1$, we distinguish the following cases:

Case 1.: $m \in\left(\frac{4}{3}, \infty\right)$. The quadratic equation (3.5) has only complex solutions, given by the expressions

$$
w_{2,3}=-\frac{1}{2} \pm i \frac{\sqrt{3 m^{2}-4 m}}{2 m} .
$$

Of course $w_{3}=\overline{w_{2}}$ (by $\bar{w}$ we denote the conjugate complex number for $w)$. 
It follows that $w_{2} w_{3}=\frac{m-1}{m}$ and $w_{2}+w_{3}=-1$. Also, using (2.2), we see that $\rho=\sqrt{\frac{m-1}{m}}<1$

Now, using (2.3), we can write that

$$
x_{n}=A_{1}(1)^{n}+A_{2}\left(w_{2}\right)^{n}+A_{3}\left(\overline{w_{2}}\right)^{n},
$$

with $A_{1}, A_{2}, A_{3}$ being real numbers. Applying Lemma 1 , it follows that

$$
\lim _{n \rightarrow \infty} x_{n}=A_{1}
$$

To find the exact value of $A_{1}$ for the considered sequences $\left(x_{n}\right)$ and $\left(y_{n}\right)$, we make the following computations:

Case 1.1.: For the sequence $\left(x_{n}\right)$.

Using (3.7) we have:

$$
\begin{aligned}
& x_{0}=0, \quad \text { so } \quad A_{1}+A_{2}+A_{3}=0 ; \\
& x_{1}=a, \quad \text { so } \quad A_{1}+w_{2} A_{2}+\overline{w_{2}} A_{3}=a ; \\
& x_{2}=b, \quad \text { so } \quad A_{1}+w_{2}^{2} A_{2}+{\overline{w_{2}}}^{2} A_{3}=b .
\end{aligned}
$$

Solving the above system for $A_{1}, A_{2}$ and $A_{3}$, we get that

$$
A_{1}=\frac{b-a\left(w_{2}+\overline{w_{2}}\right)}{w_{2} \overline{w_{2}}-\left(w_{2}+\overline{w_{2}}\right)+1},
$$

such that we have

$$
\lim _{n \rightarrow \infty} x_{n}=\frac{m(a+b)}{3 m-1} .
$$

We denote this limit by $l_{1}(m)$.

Case 1.2.: For the sequence $\left(y_{n}\right)$.

Using (3.7) we have:

$$
\begin{aligned}
& y_{0}=0, \quad \text { so } \quad A_{1}+A_{2}+A_{3}=0 \\
& y_{1}=a, \quad \text { so } \quad A_{1}+w_{2} A_{2}+\overline{w_{2}} A_{3}=0 ; \\
& y_{2}=b, \quad \text { so } \quad A_{1}+w_{2}^{2} A_{2}+{\overline{w_{2}}}^{2} A_{3}=t .
\end{aligned}
$$

Solving the above system of equations for $A_{1}, A_{2}$ and $A_{3}$, we get that

$$
A_{1}=\frac{t}{w_{2} \overline{w_{2}}-\left(w_{2}+\overline{w_{2}}\right)+1},
$$




$$
\text { "tmcs-agut" — 2010/11/14 — 22:36 — page 295 — \#9 }
$$

such that we have

$$
\lim _{n \rightarrow \infty} y_{n}=\frac{m t}{3 m-1}
$$

We denote this limit by $h_{1}(m)$.

The point in the coordinate plane with the coordinates $l_{1}(m)$ and $h_{1}(m)$ we will denote by

for any $m \in\left(\frac{4}{3}, \infty\right)$.

$$
L_{1}\left(\frac{m(a+b)}{3 m-1}, \frac{m t}{3 m-1}\right) \text {, }
$$

Thus, we have obtained that, using the present construction, the sequence of affixes $\left(z_{n}\right)$ converges, for $m \in\left(\frac{4}{3}, \infty\right)$, to a limit point $L_{1}$.

Case 2.: $m \in\left(1, \frac{4}{3}\right]$, The quadratic equation (3.5) has only real solutions, given by the expressions

$$
w_{2,3}=-\frac{1}{2} \pm \frac{\sqrt{4 m-3 m^{2}}}{2 m} .
$$

In this case it is easy to see that $-1<w_{2,3}<1$. Moreover, $w_{2} w_{3}=\frac{m-1}{m}$ and $w_{2}+w_{3}=-1$.

Using (2.3), we have

$$
x_{n}=A_{1}(1)^{n}+A_{2}\left(w_{2}\right)^{n}+A_{3}\left(w_{3}\right)^{n},
$$

with $A_{1}, A_{2}, A_{3}$ being real numbers. Applying Lemma 2, it yields $\lim _{n \rightarrow \infty} x_{n}=$ $A_{1}$.

For each of the sequences $\left(x_{n}\right)$ and $\left(y_{n}\right)$ we can find the exact value of $A_{1}$.

Case 2.1.: For the sequence $\left(x_{n}\right)$.

Using (3.7) we have:

$$
\begin{aligned}
& x_{0}=0, \quad \text { so } \quad A_{1}+A_{2}+A_{3}=0 ; \\
& x_{1}=a, \quad \text { so } \quad A_{1}+w_{2} A_{2}+w_{3} A_{3}=a ; \\
& x_{2}=b, \quad \text { so } \quad A_{1}+w_{2}^{2} A_{2}+w_{3}^{2} A_{3}=b .
\end{aligned}
$$

Solving the above system for $A_{1}, A_{2}$ and $A_{3}$, we get that

$$
A_{1}=\frac{b-a\left(w_{3}+w_{3}\right)}{w_{3} w_{3}-\left(w_{2}+w_{3}\right)+1},
$$




$$
\text { "tmcs-agut" — 2010/11/14 — 22:36 — page 296 — \#10 }
$$

such that we have

$$
\lim _{n \rightarrow \infty} x_{n}=\frac{m(a+b)}{3 m-1} .
$$

We denote this limit by $l_{1}(m)$.

Case 2.2.: For the sequence $\left(y_{n}\right)$.

Using (3.7) we have:

$$
\begin{aligned}
& y_{0}=0, \quad \text { so } \quad A_{1}+A_{2}+A_{3}=0 \\
& y_{1}=a, \quad \text { so } \quad A_{1}+w_{2} A_{2}+w_{3} A_{3}=0 \\
& y_{2}=b, \quad \text { so } A_{1}+w_{2}^{2} A_{2}+w_{3}^{2} A_{3}=t .
\end{aligned}
$$

Solving the above system for $A_{1}, A_{2}$ and $A_{3}$, we get that

$$
A_{1}=\frac{t}{w_{2} w_{3}-\left(w_{2}+w_{3}\right)+1},
$$

such that we have

$$
\lim _{n \rightarrow \infty} y_{n}=\frac{m t}{3 m-1}
$$

We denote this limit by $h_{1}(m)$.

The point in the coordinate plane with the coordinates $l_{1}(m)$ and $h_{1}(m)$ we will denote by

$$
H_{1}\left(\frac{m(a+b)}{3 m-1}, \frac{m t}{3 m-1}\right)
$$

for any $m \in\left(1, \frac{4}{3}\right]$.

Using the above construction, we have obtained that the sequence of affixes $\left(z_{n}\right)$ converges for $m \in\left(1, \frac{4}{3}\right]$ to a limit point $H_{1}$.

Comparing (3.11) and (3.16), we may conclude the following generalization

Proposition 1. In a Cartesian System we consider three given points $Z_{0}(0,0), Z_{1}(a, 0)$ and $Z_{2}(b, t)$. We build a sequence of points, denoted by $\left(Z_{n}\right)_{n \geq 0}$, such that the point $Z_{n} \in\left(Z_{n-3} Z_{n-2}\right)$ with the property $\frac{\left|Z_{n-3} Z_{n}\right|}{\left|Z_{n-3} Z_{n-2}\right|}=\frac{1}{m}$ (where $m>1$ ) for any $n \geq 0$, having the starting point $Z_{2}$. This sequence of points converges and has as a limit point, the point $\Gamma$ with the coordinates $\Gamma\left(\frac{m(a+b)}{3 m-1}, \frac{m t}{3 m-1}\right)$. 


$$
\text { "tmcs-agut" — 2010/11/14 — 22:36 — page } 297 \text { — \#11 }
$$

\subsection{The specialization}

With the choice $m=2$ the solution of Problem 1 can be obtained:

Proposition 2. In an arbitrary triangle $A B C$ we consider the midpoints $M_{1} \in(A B), M_{2} \in(B C), M_{3} \in\left(C M_{1}\right), M_{4} \in\left(M_{1} M_{2}\right)$, etc. of the line segments $(A B),(B C),\left(C M_{1}\right),\left(M_{1} M_{2}\right)$, etc., respectively. If we continue to build such points $M_{n}$, then for $n \rightarrow \infty$, the point $M_{n}$ will have the coordinates $\left(\frac{2(a+b)}{5}, \frac{2 t}{5}\right)$, where $A(0,0), B(a, 0)$ and $C(b, t)$.

\section{Exploring the properties of limit-points}

\subsection{Other limit points}

In the previous section we have considered $Z_{2}$ be the starting point for the sequence $\left(Z_{n}\right)$. In this section we will consider the rest of the two cases (getting from cyclic permutation of the vertices of the triangle) for starting points, namely $Z_{0}$ and $Z_{1}$, respectively.

To keep the form of the work and for simplicity, we will permute the notations of the vertices, keeping the coordinates, so that: $Z_{0}^{\prime} Z_{1}^{\prime} Z_{2}^{\prime}$ is a triangle with the vertices $Z_{0}^{\prime}(b, t), Z_{1}^{\prime}(0,0)$ and $Z_{2}^{\prime}(a, 0)$, where $a>0, b>0, t>0$ (for convenience $a>b)$ are real numbers.

We consider the starting point to be $Z_{2}^{\prime}$, i.e. we choose a point, namely $Z_{3}^{\prime} \in$ $\left(Z_{0}^{\prime} Z_{1}^{\prime}\right)$ such that $\frac{\left|Z_{0}^{\prime} Z_{3}^{\prime}\right|}{\left|Z_{0}^{\prime} Z_{1}^{\prime}\right|}=\frac{1}{m}, m>1$. We continue the same process developed in the previous section and after all the correspondent computations we may state:

Proposition 3. In a Cartesian System we consider three given points $Z_{0}^{\prime}(b, t), Z_{1}^{\prime}(0,0)$ and $Z_{2}^{\prime}(a, 0)$. We build a sequence of points, denoted by $\left(Z_{n}^{\prime}\right)_{n \geq 0}$, such that the point $Z_{n}^{\prime} \in\left(Z_{n-3}^{\prime} Z_{n-2}^{\prime}\right)$ with the property $\frac{\left|Z_{n-3}^{\prime} Z_{n}^{\prime}\right|}{\left|Z_{n-3}^{\prime} Z_{n-2}^{\prime}\right|}=\frac{1}{m}$ (where $m>1$ ) for any $n \geq 0$, having the starting point $Z_{2}^{\prime}$. This sequence of points converges and has as a limit point, the point $\Omega$ with the coordinates $\Omega\left(\frac{a(m-1)+m b}{3 m-1}, \frac{m t}{3 m-1}\right)$.

For the last situation, we will permute again the names of the vertices of the given triangle, such that we obtain $Z_{0}^{\prime \prime} Z_{1}^{\prime \prime} Z_{2}^{\prime \prime}$ with the vertices $Z_{0}^{\prime \prime}(a, 0), Z_{1}^{\prime \prime}(b, t)$ and $Z_{2}^{\prime \prime}(0,0)$, where $a>0, b>0, t>0$ (for convenience $a>b$ ) are real numbers.

We consider the starting point to be $Z_{2}^{\prime \prime}$, i.e. we choose a point, namely $Z_{3}^{\prime \prime} \in\left(Z_{0}^{\prime \prime} Z_{1}^{\prime \prime}\right)$ such that $\frac{\left|Z_{0}^{\prime \prime} Z_{3}^{\prime \prime}\right|}{\left|Z_{0}^{\prime \prime} Z_{1}^{\prime \prime}\right|}=\frac{1}{m}, m>1$. We continue the same process 


$$
\text { "tmcs-agut" — 2010/11/14 — 22:36 — page 298 — \#12 }
$$

developed in the previous section and after all the correspondent computations we may state:

Proposition 4. In a Cartesian System we consider three given points $Z_{0}^{\prime \prime}(a, 0), Z_{1}^{\prime \prime}(b, t)$ and $Z_{2}^{\prime \prime}(0,0)$. We build a sequence of points, denoted by $\left(Z_{n}^{\prime \prime}\right)_{n \geq 0}$, such that the point $Z_{n}^{\prime \prime} \in\left(Z_{n-3}^{\prime \prime} Z_{n-2}^{\prime \prime}\right)$ with the property $\frac{\left|Z_{n-3}^{\prime \prime} Z_{n}^{\prime \prime}\right|}{\left|Z_{n-3}^{\prime \prime} Z_{n-2}^{\prime \prime}\right|}=\frac{1}{m}$ (where $m>1$ ) for any $n \geq 0$, having the starting point $Z_{2}^{\prime \prime}$. This sequence of points converges and has as a limit point, the point $\Sigma$ with the coordinates $\Sigma\left(\frac{b(m-1)+m a}{3 m-1}, \frac{(m-1) t}{3 m-1}\right)$.

REMARK 1. As a conclusion about the above construction, for any choice of starting the sequence, the limit of it is one of the points $\Gamma, \Omega$ or $\Sigma$, called the limit points.

\subsection{The connection between the triangles $\triangle A B C$ and $\Delta \Gamma \Omega \Sigma$}

In this part we will find connections between the triangles $\triangle A B C$ and $\triangle \Gamma \Omega \Sigma$.

We denote the vertices of the given triangle in this way: $A=Z_{0}(0,0), B=$ $Z_{1}(a, 0)$ and $C=Z_{2}(b, t)$. Also, we denote by $M, N, P$ the midpoints of the segments $(A B),(B C)$ and $(C A)$, respectively. From (2.5) we find the coordinates $M\left(\frac{a}{2}, 0\right), N\left(\frac{a+b}{2}, \frac{t}{2}\right)$ and $P\left(\frac{b}{2}, \frac{t}{2}\right)$.

It is well known ([7]) that the lines $A N, B P$ and $C M$ will intercept in the weight (mass) center $G$ of the triangle $A B C$, with the coordinates:

$$
G\left(\frac{a+b}{3}, \frac{t}{3}\right)
$$

Immediately we may find the equations of the medians:

$$
\begin{aligned}
A N: y & =\frac{t}{a+b} x \\
B P: y & =\frac{t}{b-2 a} x-\frac{a t}{b-2 a} \\
C M: y & =\frac{2 t}{2 b-a} x-\frac{a t}{2 b-a} .
\end{aligned}
$$

Now we will make some connections between the limit points $\Gamma, \Omega$ and $\Sigma$, and the triangle $A B C$.

First we have the following 


$$
\text { "tmcs-agut" — 2010/11/14 — 22:36 — page 299 — \#13 }
$$

Proposition 5. If the triangle has the vertices $A(0,0), B(a, 0)$ and $C(b, t)$ and $M, N, P$ the midpoints of the segments $(A B),(B C)$ and $(C A)$, respectively, then the following occur:
(a) $\Gamma \in A N$;
(b) $\Omega \in B P$;
(c) $\Sigma \in C M$.

Moreover, we have the following slopes for the given triangle's sides:

$$
A B: m_{A B}=0 ; \quad B C: m_{B C}=\frac{t}{a-b} ; \quad A C: m_{A C}=\frac{t}{b},
$$

and also for the lines:

$$
\Gamma \Omega: m_{\Gamma \Omega}=0 ; \quad \Omega \Sigma: m_{\Omega \Sigma}=\frac{t}{a-b} ; \quad \Gamma \Sigma: m_{\Gamma \Sigma}=\frac{t}{b},
$$

such that it follows:

Proposition 6. Let us consider the triangle $\triangle A B C$, with the coordinates $A(0,0), B(a, 0)$ and $C(b, t)$. Furthermore, let $\Gamma, \Omega$ and $\Sigma$ be the limit points constructed in the above three processes. The the triangles $\triangle A B C$ and $\triangle \Gamma \Omega \Sigma$ are similar.

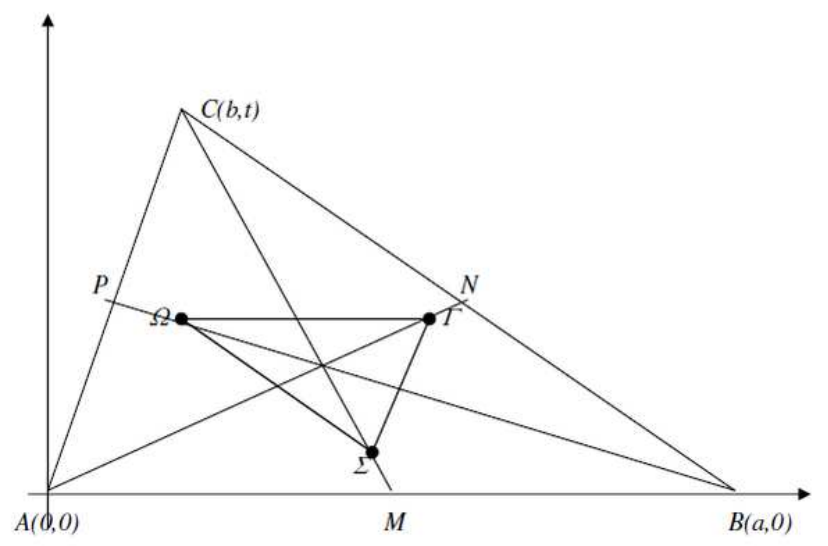

Figure 3 


$$
\text { "tmcs-agut" — 2010/11/14 — 22:36 — page 300 — \#14 }
$$

\subsection{Boundaries for limit points}

In the last part of the section we will observe few interesting analytical properties of the limit-points.

We know so far that for $m \in(1, \infty)$ the limit-points are $\Gamma\left(\frac{m(a+b)}{3 m-1}, \frac{m t}{3 m-1}\right)$, $\Omega\left(\frac{a(m-1)+m b}{3 m-1}, \frac{m t}{3 m-1}\right)$ and $\Sigma\left(\frac{b(m-1)+m a}{3 m-1}, \frac{(m-1) t}{3 m-1}\right)$ (depending on how we start the construction of the sequence of points) and also that $\Gamma \in A N, \Omega \in B P$, and $\Sigma \in C M$.

Our question is if, for example, $\Gamma \in(A N)$, i.e. can lie on any position of the median $A N$. To study this, we take into consideration the coordinates of the limit points. We see that all of them depend continuously on $m$ (where $m>1$ ).

For this reason we are able to build the following continuous functions (over their domains):

$$
\begin{aligned}
& f_{1}(m)=\frac{m(a+b)}{3 m-1}, \quad \text { and } \quad g_{1}(m)=\frac{m t}{3 m-1} \\
& f_{2}(m)=\frac{a(m-1)+m b}{3 m-1}, \quad \text { and } \quad g_{2}(m)=\frac{m t}{3 m-1} \\
& f_{3}(m)=\frac{b(m-1)+m a}{3 m-1}, \quad \text { and } \quad g_{3}(m)=\frac{(m-1) t}{3 m-1} .
\end{aligned}
$$

Moreover, for fixed values of $a, b$ and $t$, we obtain that:

$f_{1}^{\prime}(m)<0$ and $g_{1}^{\prime}(m)<0$ for any $m>1$, such that $f_{1}$ and $g_{1}$ are strictly monotone functions;

$f_{2}^{\prime}(m)>0$ and $g_{2}^{\prime}(m)<0$ for any $m>1$, such that $f_{1}$ and $g_{1}$ are strictly monotone functions;

$f_{3}^{\prime}(m)>0$ for $a>2 b$ or $f_{3}^{\prime}(m) \leq 0$ for $a \leq 2 b$ and $g_{3}^{\prime}(m)>0$ for any $m>1$, such that $f_{1}$ and $g_{1}$ are monotone functions.

Let us take the limits as follows:

$$
\begin{array}{ll}
\lim _{m \rightarrow 1} \frac{m(a+b)}{3 m-1}=\frac{a+b}{2} ; & \lim _{m \rightarrow \infty} \frac{m(a+b)}{3 m-1}=\frac{a+b}{3} \\
\lim _{m \rightarrow 1} \frac{m t}{3 m-1}=\frac{t}{2} ; & \lim _{m \rightarrow \infty} \frac{m t}{3 m-1}=\frac{t}{3} \\
\lim _{m \rightarrow 1} \frac{a(m-1)+m b}{3 m-1}=\frac{b}{2} ; & \lim _{m \rightarrow \infty} \frac{a(m-1)+m b}{3 m-1}=\frac{a+b}{3} \\
\lim _{m \rightarrow 1} \frac{m t}{3 m-1}=\frac{t}{2} ; & \lim _{m \rightarrow \infty} \frac{m t}{3 m-1}=\frac{t}{3} \\
\lim _{m \rightarrow 1} \frac{b(m-1)+m a}{3 m-1}=\frac{a}{2} ; & \lim _{m \rightarrow \infty} \frac{b(m-1)+m a}{3 m-1}=\frac{a+b}{3}
\end{array}
$$




$$
\text { "tmcs-agut" — 2010/11/14 — 22:36 — page 301 — \#15 }
$$

$$
\lim _{m \rightarrow 1} \frac{(m-1) t}{3 m-1}=0 ; \quad \quad \lim _{m \rightarrow \infty} \frac{(m-1) t}{3 m-1}=\frac{t}{3} .
$$

In this situation, we are able to state the following

Proposition 7. The limit-points $\Gamma, \Omega$ and $\Sigma$ belong continuously to the medians $A N, B P$, and $C M$, respectively, between the points as follows:

(a) $\Gamma \in A N$, between $G\left(\frac{a+b}{3}, \frac{t}{3}\right)$ and $N\left(\frac{a+b}{2}, \frac{t}{2}\right)$;

(b) $\Omega \in B P$, between $G\left(\frac{a+b}{3}, \frac{t}{3}\right)$ and $P\left(\frac{b}{2}, \frac{t}{2}\right)$;

(c) $\Sigma \in C M$, between $G\left(\frac{a+b}{3}, \frac{t}{3}\right)$ and $M\left(\frac{a}{2}, 0\right)$.

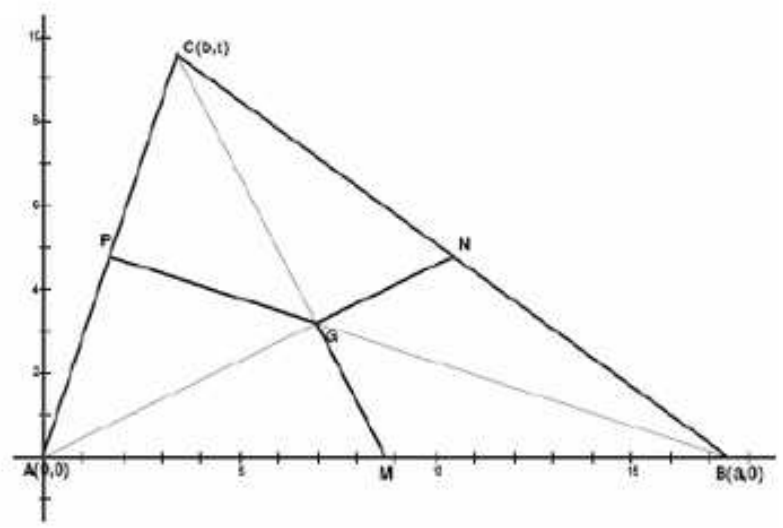

Figure 4

\section{References}

[1] M. Cocuz, Culegere de Probleme de Matematica (Mathematics Problems), Editura Academiei RSR, Bucuresti, 1984 (in Romanian).

[2] C. Jordan, Calculus of finite differences, Third Edition, Carver Chelsea Publishing Co., New York, 1965.

[3] R. Larson, R. P. Hostetler and B. H. Edwards, Calculus with Analytic Geometry, Eight Edition, Houghton Mifflin Comp, Boston, New York, 2006.

[4] J. E. Marsden and M. J. Hoffman, Basic Complex Analysis, (W. H. Freeman \& Company; Third edition, eds.), 1999. 


$$
\text { "tmcs-agut" — 2010/11/14 — 22:36 — page 302 — \#16 }
$$

[5] K. S. Miller, An introduction to the calculus of finite differences and difference equations, Dover Publications, Inc., New York, 1966.

[6] L. M. Milne-Thompson, The Calculus of Finite Differences, Macmillan and Co., Ltd., London, 1951.

[7] E. Moise, Elementary Geometry from an Advanced Standpoint, Third Ed., Addison Wesley, 1990.

[8] N. E. Nörlund, Differenzenrechnung, Springer, Berlin, 1926.

CALIN M. AGUT and IOANA E. AGUT

BRAZOSPORT COLLEGE

500 COLLEGE DRIVE

LAKE JACKSON, TX 77566

USA

E-mail: Calin.Agut@brazosport.edu

E-mail: Ioana.Agut@brazosport.edu

(Received May, 2010) 